\title{
Ion Cloud Manipulation Using the Radiofrequency-Only-Mode as an Improvement for High Mass Detection in Fourier Transform Mass Spectrometry
}

\author{
C. B. Jacoby, C. L. Holliman, D. L. Rempel, and M. L. Gross \\ Midwest Center for Mass Spectrometry, Department of Chemistry, University of Nebraska, Lincoln, Nebraska, \\ USA
}

\begin{abstract}
It has been difficult to achieve the expected high resolving power for high-mass biomolecule ions in Fourier transform mass spectrometry. Our hypothesis is that ion clouds produced by laser desorption or injection are diffuse and produce poor signals. To test the hypothesis, clouds of benzene molecular ions produced by electron ionization were purposefully expanded via magnetron mode excitation and characterized by a new experimental sequence for cloud sectional analysis. The expanded cloud was then successfully focused to the trap center by using a high-pressure dynamic event (radiofrequency-only mode). The expanded cloud in a conventional cubic trap produces no detectable signal, whereas the focused cloud in a compensated trap yields a high-resolution signal with good signal-to-noise ratio. ( $T$ Am Soc Mass Spectrom 1993, 4, 186-189)
\end{abstract}

$\mathrm{T}$ The tools for the reduction of radial ion cloud distribution size in Fourier transform mass spectrometry (FTMS) are surprisingly limited. This is particularly a problem because the method derives its primary advantages from the confinement of ions for long periods of time. Marshall et al. [1] showed that it is possible to reduce the cyclotron radius of the cloud by application of a resonant excitation waveform that is $180^{\circ}$ out of phase with respect to the ion cloud. This is effective only if the state of the ion cloud has not been severely degraded or dephased via ion-neutral collisions, coulombic forces, or electric field inhomogeneities. Another approach is modulation of electric fields in a Penning trap to couple the magnetron mode to other modes (e.g., axial or cyclotron), as was originally recognized and demonstrated by Dehmelt and co-workers [2, 3]. In 1986, Brown and Gabrielse [4] reviewed the subject in detail. This review led to recent applications $[5,6]$ of motional sideband "cooling" of the magnetron motion prior to making accurate mass measurements of radionuclide ions injected into a Penning trap from an isotope separator.

Important ionization methods in chemistry, such as matrix-assisted laser desorption (MALD), electrospray ionization, and fast-atom bombardment or liquid secondary ionization mass spectrometry, can produce massive ions with kinetic energy distributions that are

Address reprint requests to Michael L. Gross, Department of Chemistry, University of Nebraska, Lincoln, NE 68588-0304. high compared with the normal trapping well depths of a few volts [7-11]. Energetic ions can be trapped in deeper wells $(>10 \mathrm{~V})$, but these result in poor performance owing to larger electric field inhomogeneities. Approaches to compress the axial energy distributions by using electric and magnetic field manipulations in the absence of collisions must result in increased radial cloud size because these methods are conservative and the phase space volume of the cloud distribution is constant, as required by Liouville's theorem [12].

Smalley and co-workers [13] and Solouki and Russell [14] demonstrated translational dampening via nonconservative methods by cooling the ions energies in a "waiting room." This approach allows for the use of more moderate trapping wells, and detection performance is improved. Marshall and co-workers [15] take another approach by applying the motional sideband "cooling" methods mentioned above to "shrink-wrap" ion clouds in chemistry experiments.

In this report a different approach, namely, the incorporation of a temporary dynamic trapping mode event [radiofrequency ( $r f$ )-only mode event] into the FTMS sequence, is used to shape the ion cloud so that subsequent excitation and detection are improved. We show that the rf-only mode event focuses a broadly distributed ion cloud to the center of the trap. When combined with trap electric field compensation [16-18], the use of the rf-only mode substantially improves the detection of ions that are initially located away from the trap center. 
The rf-only mode in the FTMS sequence draws on the work of Bonner et al. [19] with the quadrupolar ion storage device, or QUISTOR. They showed that at pressures considerably higher than are appropriate for FTMS, collisions with helium neutral molecules focus ions to the center of the trap. In preliminary announcements, we demonstrated not only that this active trapping mode can be applied to FTMS, but also that transient adducts in ion-molecule reactions can be collisionally stabilized at high pressures in an FTMS trap [20-24]. This latter application cannot be accommodated by motional sideband "cooling" because the applied pressure is too low.

\section{Experimental}

A compensated trap, in which the outer segment can be used for ion quenching, and an rf-only mode event were used for the experiments reported here. The normal trap plates of a $25.4-\mathrm{mm}$ cubic trap at $1.2 \mathrm{~T}$ were replaced with a set of segmented trap plates consisting of a disk of 6.35 -mm radius separated by approximately $0.254 \mathrm{~mm}$ from the outer segment. The two segments of each trap plate may be held at the same potential $(1.0 \mathrm{~V})$ for normal FTMS operations or held at different potentials as in the case of a compensated trap (1.0 V on the disks and $1.7 \mathrm{~V}$ on the outer segments). These segmented trap plates also allow any portion of the ion cloud that has been excited to a radius greater than $6.35 \mathrm{~mm}$ to be quenched from the trap by applying a potential of $-1.0 \mathrm{~V}$ on the outer segment of the plates.

Benzene at a pressure of approximately $1 \times 10^{-7}$ torr was ionized along the $z$-axis by a $20-\mathrm{eV}$ electron beam of $100 \mathrm{nA}$ for a period of approximately $15 \mathrm{~ms}$. A 2-s delay after ionization was used in all experiments to allow the $z$-mode amplitudes to dampen before any additional operations were executed in the sequence.

The rf-only mode event was implemented by modulating the segmented trap plates with a $800-\mathrm{V}, 1.3-\mathrm{MHz}$ sine wave for $4 \mathrm{~s}$ during the delay interval of the sequence. During the first second in which the rf event was active, the cell was pressurized with helium to approximately $3 \times 10^{-3}$ torr through a pulsed Varian variable leak valve (model 951-5100; Varian Associates, California). A 3-s delay was implemented after the if event so that nearly all of the neutral helium was removed.

\section{Results and Discussion}

Imperative to this study is the ability to control and probe the radial size or cross section of an ion cloud. After forming a tight ion cloud by electron ionization (EI), the cloud was expanded radially (via magnetron excitation) to mimic the outer perimeter of the diffuse clouds that are formed by desorption ionization. When appropriate, the rf-only mode event was then applied to focus the ion cloud back to the cell center. The radial extent of the resulting cloud was obtained in a sequence of measurements. The cloud was incrementally displaced from its center by using a second magnetron excitation event, and the ions that remained after quenching the outer portion of the cloud were detected.

The displacement was accomplished with a second $758-\mathrm{Hz}, 0.170-\mathrm{V}$ magnetron mode excitation that was applied $180^{\circ}$ out of phase to each of the two excitation plates of a compensated trap. A quench of the outer segment was then applied to rid the cell of any ions with a resulting magnetron radius greater than the radius of the disk electrode. The remaining ions were excited via a $250-\mu s, 0.68-\mathrm{V}$ rf burst at the resonant cyclotron frequency and then detected for a relatively short $0.208 \mathrm{~ms}$. A curve characterizing the cross section was obtained by plotting remaining ion numbers, measured as peak complex areas, as a function of the second magnetron excitation time. A cloud with a small magnetron radius distribution is expected to produce a level, high-amplitude complex area followed by a relatively sharp decrease to noise. On the other hand, a cloud with a broad distribution is expected to produce a more gradual decrease in complex area, starting at shorter magnetron excitation times and persisting for longer times.

The successful sectional analysis of the cloud can be seen in Figure 1. After ion formation, the cloud was expanded via the first magnetron mode excitation to approximately $50 \%$ of the cell dimension. The rf-only mode or an equivalent time delay was applied to give the curves shown in Figure 1. The cross-section curve without the rf event shows that the initial signal is low as a result of the majority of the ions having magnetron radii beyond the radius of the disk and being quenched from the cell (as depicted schematically in Figure $\left.2 \mathbf{a}, t_{1}\right)$. As the second magnetron excitation time is increased and the ion cloud is displaced, it is apparent that a portion of the ion cloud is brought within the confines of the disk electrode (Figure $2 a, t_{2}$ ) and therefore not removed from the cell. At second excitation times longer than $3.5 \mathrm{~ms}$, the ion cloud in its entirety is translated to a position where nearly complete quenching from the cell was observed (Figure $2 a, t_{3}$ ).

The successful focusing of an expanded ion cloud by the rf-only mode is shown by the dashed line in Figure 1. Evidence for success is the level complex area at short excitation times followed by a comparatively rapid decline in complex area as the ion cloud is translated beyond the radius of the disk at $2.125 \mathrm{~ms}$. The focused cloud remains entirely within the confines of the disk as the second magnetron excitation is increased (Figure $2 b, t_{1}^{\prime}$ and $t_{2}^{\prime}$ ) and then moves rapidly to the region where it can be quenched from the cell (Figure 2b, $t_{3}^{\prime}$ ). The cross-section curve (not shown) of an undisturbed ion cloud formed by EI is nearly identical to that of a cloud expanded to approximately $50 \%$ of the cell and subject to the rf event. 


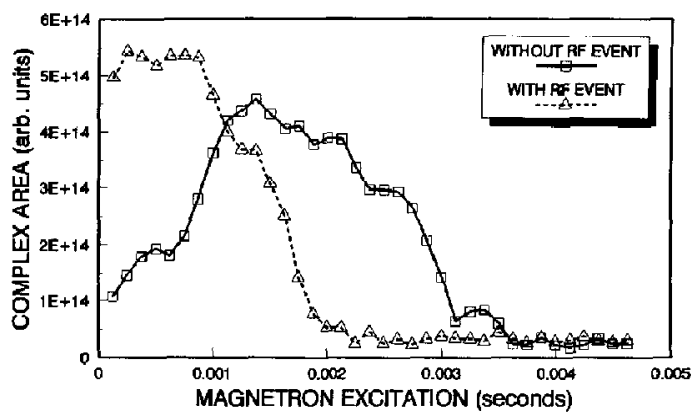

Figure 1. Cross-section curve for an ion cloud formed by EI and expanded to a magnetron radius of approximately $50 \%$ of the cell size; (-) unfocused ion cloud whose signal increases and decreases with time owing to the translation of the ion cloud to a radii greater than and less than that of the disk electrode; $(-)$ ion cloud focused by an rf event whose signal starts level and drops off as the cloud is translated to a radii greater than that of the disk electrode.

A test of the efficacy of compensation and the rf-only mode event to improve mass spectra is shown by the four spectra in Figure 3a-d. In all cases, the initial ion clouds, which were formed by identical ionization events, were expanded to approximately $50 \%$ of the cell size, as bcfore. Figure $3 a$, for which compensation and $\mathrm{rf}$ events were not used, shows no detectable signal (the signal seen at $m / z 77.75$ is an electronic noise spike). Figure $3 b$ shows that with only the use of the $r f$ event, the ion cloud is focused to yield a marginal signal with a signal-to-noise ratio of about $2: 1$. In Figure 3c, with only the use of compensation, no detectable signal is seen. The use of both the rf event and compensation (Figure $3 \mathrm{~d}$ ) gives a signal representing successful focusing via the rf event. The improve-
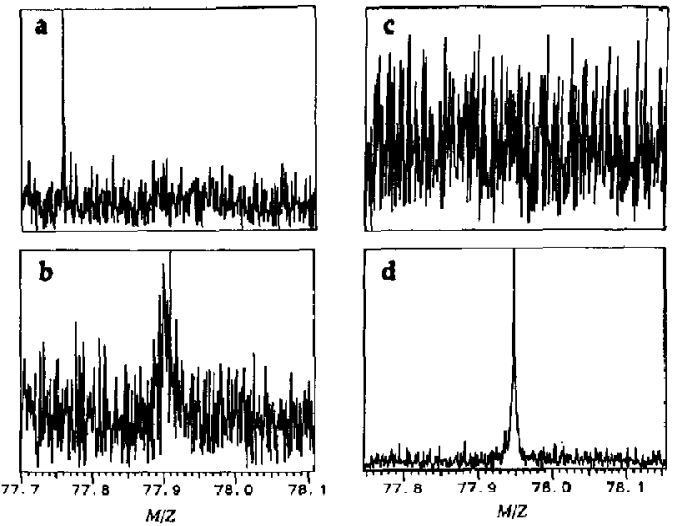

Figure 3. Mass spectra of a $1.25-\mathrm{kHz}$ region of the mass range for the molecular ion of benzene for an ion cloud translated to approximately $50 \%$ of the cell size under different conditions of compensation and if events: (a) no compensation or if event; (b) only an if event; (c) only compensation applied; (d) both compensation and if event applied.

ment is a peak with a signal-to-noise ratio of approximately 18 and a resolution of $32.5 \mathrm{~K}$ (full-width half-maximum).

In this report, we have demonstrated a method that reduces the radial size of an ion cloud. Because the cross-section curves are nearly identical for ion clouds formed by EI and for those expanded to $50 \%$ of the cell dimension, we suggest that the ion cloud shape after the $\mathrm{rf}$ event is independent of the shape prior to the event. With this method, it may be possible to tune the instrument with an ion cloud produced in a controlled way by EI and then analyze ions produced in diffuse clouds (e.g., by MALD or injection) without needing to change the excitation and detection parameters, thus
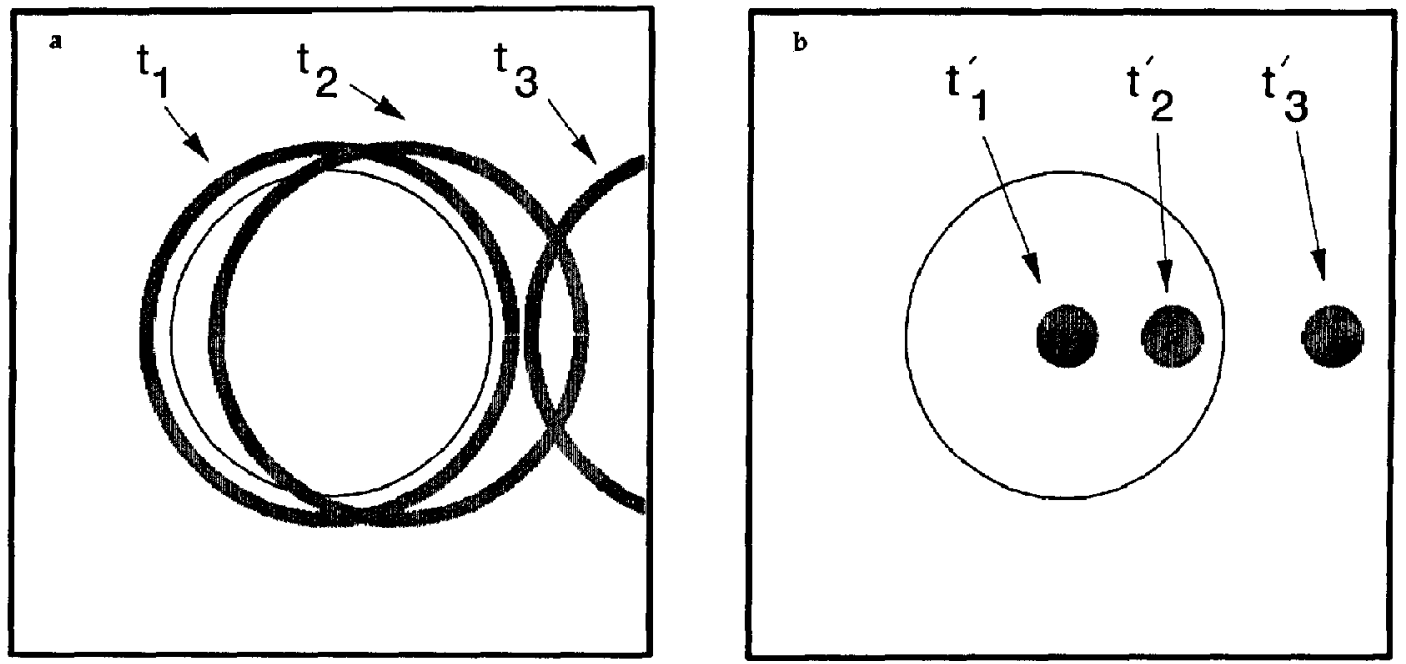

Figure 2. Schematic view of expanded (a) and compact (b) ion clouds as seen against the backdrop of a compensated cell trap plate. Displacement of the ion cloud is accomplished by applying the second magnetron excitation for longer times, $t_{1}<t_{2}<t_{3}$ and $t_{1}^{\prime}<t_{2}^{\prime}<t_{3}^{\prime}$. Quenching of the compact cloud onto the outer trap electrode occurs more sharply than for the expanded cloud. 
standardizing the operation of the instrument. We also propose that with the use of the rf-only mode, the high kinetic energy of ions produced, for example, by MALD, can be removed by collisions with pulsed helium within the interior of the trap, providing improved overall efficiency.

\section{Acknowledgment}

This work was supported by National Science Foundation grant CHE-9017250.

\section{References}

1. Marshall, A. G.: Lin Wang, T.-C.; Lebahuan Ricra, T. Chem. Phys. Lett. 1984, 105(2), 233-236.

2. Wineland, D.; Dehmelt, H. Int. J. Mass Spectrom. Ion Processes 1975, 16, 338-342.

3. Van Dyck, R. S. Jr.; Schwinberg, P. B.; Dehmelt, H. G. In New Frontiers in High Energy Physics; Kursunoglus, B.; Perlmutter, A.; Scott, L., Eds,; Plenum: New York, 1978.

4. Brown, L. S.; Gabrielse, G. Rev. Modern Phys. 1986, 58(1), 258-267.

5. Bollen, G.; Moore, R. B.; Savard, G.; Stolzenberg, H. J. Appl. Phys. 1990, 68(9), 4355-4374.

6. Savard, G.; Becker, S.; Bollen, G.; Kluge, H.-J.; Moore, R. B.; Otto, T.; Schweikhard, L.; Stolzenberg, H.; Wiess, U. Phys. Lett. A 1991, 158(5), 247-252.

7. Hardin, E. D.; Vestal, M. L. Attal. Chem. 1983, 53, 1492-1497.

8. Van der Peyl, G. J. Q.; Van der Zande, W. J.; Bederski, K.; Boerboom, A. J. H.; Kistemaker, P. G. Int. I. Mass Spectrom. Ion Processes 1983, 47, 7-10.

9. Yang, M.; Reilly, J. P. J. Phys. Chem. 1990, 94, 6299-6305.

10. Pan, Y.; Cotter, R. J. Proceedings of the 39th ASMS Conference on Mass Spectrometry and Allied Topics; Nashville, TN, May 1991.

11. Beavis, R. C.; Chait, B. T. Chem. Phys. Lett. 1991, 181(5), 479-484.

12. Arnold, V. I., Ed. Mathematical Methods of Classical Mechanics, 2nd ed., Graduate Texts in Mathematics; Springer-Verlag: New York.

13. Maruyma, S.; Anderson, L. R.; Smalley, R. E. Rev. Sci. Instrum. 1990, 61, 3686-3693.

14. Soluoki, T.; Russell, D. H. Proc. Natl. Acad. Sci. U.S.A., in press.

15. Guan, S.; Xiang, X.; Marshall, A. G. in press.

16. Huang, 5. K.; Rempel, D. L. Proceedings of the 34th ASMS Conference on Mass Spectrometry and Allied Topics; Cincinnati, $\mathrm{OH}, 1986$.

17. Rempel, D. L. Procedings of the 35th ASMS Conference on Mass Spectrometry and Allied Topics, Denver, CO, 1987.

18. Rempel, D. L.; Grese, R. P.; Gross, M. L. Int. I. Mass Spectram. Ion Processes 1990, 100, 381-395.

19. Bonner, R. F.; March, R. E.; Durup, J. Int. J. Mass Spectrom. Ion Processes 1976, 22, 17-34.

20. Rempel, D. L.; Gross, M. L. Proceedings of the 38th ASMS Conference on Mass Spectrometry and Allied Topics, Tucson, AZ, 1990.

21. Rempel, D. L.; Gross, M. L. Proceedings of the 39th ASMS Conference on Mass Spectrometry and Allied Topics, Nashville, TN, 1991.

22. Holliman, C. L.; Rempel, D. L.; Gross, M. L. Proceedings of the 40th ASMS Conference on Mass Spectrometry and Allied Topics, Washington, DC, 1992.

23. Yu, S. J.; Holliman, C. L., Rempel; D. L.; Gross, M. L. I. Am. Chem. Soc., submitted.

24. Rempel, D. L.; Gross, M. L. J. Am. Soc. Mass Spectrom. 1992, $3,590-594$. 